a general amelioration of the symptoms, it was repeated in about a week, removing again about two ounces of fluid, less turbid than before, still containing no micro-organisms.

Internally she took most of the time mercury and iodid of potassium. On March 1 we began collargol inunctions, used twice daily. On March 5 the clinical record showed that patient had a slight chill. In a few days, however, improvement set in and on March 8 temperature dropped to normal, and never afterward rose above 99 . The mastoid wound did well from the first, and was completely healed in about two months. The hearing in the affected ear improved and the patient rapidly returned to normal.

This case, I think, is instructive in that it is an instance of a recovery from pronounced leptomeningitis, the clinical diagnosis being confirmed by the results of the lumbar puncture. It is one more to be added to the favorable cases previously reported by Macewen, Gradenigo, Brieger and others, which encourages us not to take an absolutely hopeless view on witnessing this dread disease develop as a complication of middle-ear suppuration. While from the given case I am not able to draw any satisfying conclusions as to the cause of ultimate recovery-whether it should be attributed to the operation on the diseased mastoid, removing the focus of infection, or to the lumbar puncture, relieving intracranial pressure and diminishing the amount of septic fluid, or to the possible beneficial influence of the collargol inunctions-I believe that in a similar case one need not hesitate to use all these measures, as each is indicated and possibly all three may contribute somewhat to the ultimate recovery of the patient.

Attention is called to the comparatively insignificant character of the aural symptoms. While not duplicating the former case in the absence of any history of suppuration, the discharge was slight and of short duration and had ceased completely at the period when the intracranial symptoms developed.

In the bone involvement a strong contrast is presented with the condition noted in Case 1 , in which the bone destruction was far-reaching and profound, the dural membrane being in direct contact with diseased bone over a considerable area. In this, on the contrary, necrosis was very slight, the disease not reaching the inner table at any point.

815 Connecticut Avenue.

\section{PHOSPHATIC CALCULUS OF THE POSTERIOR URETHRA}

\section{E. F. KILBANE, M.D}

Assistant Surgeon, Roosevelt Hospital, Out-Patient Department of Genitourinary Department NEW YORK

Patient.-J. H., came to the clinic complaining of difficult urination, increased urination and decided urgency, with occasional attacks of complete retention. One such attack had occurred the day previous, for the relief of which he had been catheterized at another institution. He had had an attack of complete retention one year previously, and gave a history of several attacks of urethritis during his youth. He had been treated for stricture at one time. He had no hematuria and no pain.

Examination.-The patient was a well-preserved man about 55 years old. The urine was very cloudy and contained many long shreds. The catheter met with obstruction in the prostatic urethra. A small woven bougie passed into the bladder. A No. 10 (French scale) tunneled steel catheter guided over a whalebone filiform passed over a long soft calculus on the floor of the posterior urethra. The end of the calculus was seen through an endoscopic tube.
Operation.-Under cocain anesthesia I attempted to remove the stone through the endoscope, using a pair of long forceps with narrow blades, but succeeded only in breaking off small fragments of the stone, as the narrow caliber of the tube prevented the handle of the forceps opening to an extent sufficient to grasp the calculus firmly in the blades. As the pain became intense and hemorrhage profuse, further manipulations were not attempted at the time, since it appeared that an external urethrotomy would be necessary. Soon after arising from the table the patient felt inclination to urinate, and when he tried to do so the stream of urine forced the stone to within one inch of the external meatus, from which position it was easily removed with thumb forceps. After removal it was found to be a soft, friable, phosphatic calculus, $0.5 \mathrm{~cm}$. in diameter and $3 \mathrm{~cm}$. long, cylindrical, conforming to the shape of the posterior urethra. A size 26 steel sound was easily passed into the urethra on the following day. The patient erjoyed complete relief from all urinary symptoms afterward.

24 West Sixtieth St.

\section{TRANSFUSION OF BLOOD IN A CASE. OF PELLAGRA*}

H. P. COLE, M.D.

Instructor in Gynecology. Medical Department of the University ố Alabama MOBILE, ALA.

Pellagra, a trophoneurotic disease now appearing in this portion of the country, possesses a high mortality rate in its acute type; 64 per cent. in the cases reported by Searcy. ${ }^{\prime}$ Pellagra may probably be classed as one of the diseases caused by vegetable poisons, pellagrazein, a fatty oil or extractive so named by Lombroso. Pellagrazein is developed in fermenting and decomposing grain in the presence of a fungus. From the ingestion of this poison in corn meal the skin and nerve lesions characteristic of the disease result. As a rule there is associated with the disorder a pernicious dysentery probably of toxic origin. No cure has been found as yet.

For a more complete account of the disease the excellent articles by Bellamy, ${ }^{2}$ Searcy, ${ }^{1}$ Merrill $^{3}$ and that of Randolph presented at this meeting may be consulted.

Considering the possibility that recovery from an attack of pellagra might produce a certain degree of immunity in the patient, the use of transfusion of blood from such a recovered patient in an acute case of pellagra suggested itself, as all medical means have failed in the treatment of this disease.

It is impossible to state the nature of the immunity until the etiology of the disease is definitely ascertained. If pellagrazein is the etiologic factor, the immunity may be of the nature of the antitoxin (antiriciu) produced experimentally in lower animals against ricin.

To lend weight to any positive findings a recipient was found in such extreme condition that recovery was absolutely impossible in the opinion of one who has carefully followed all the cases of a large epidemic. To add further weight to the evidence, all medication was withdrawn after transfusion.

- Synopsis of paper read before the Southern Medical Association at Atlanta, Ga., Nor. 12, 1908

1. Searcy, George H.: An Epidemic of Acute Pellagra, IHE Jotknal A. M. A., July 6, 1907, xlix, 37.

2. Bellamy, R. Harllee: Pellagra: Its Occurrence in 'This Country: Report of Cases, The Journal A. M. A., Aug. 1, 1908, li, 397.

3. Merrill, 'T. C.: A Sporadic Case Diagnosed as Pellazra, THE Jounnal A. M. A. Sept. 14, 1907, xlix, 940.

4. Randolph, James H.: Pellagra and Pellagrins; with Report of Cases, Arch. Int. M 2 d., January, 1908 
'The patient's extreme condition might best be shown when we state that she was one of two selected to be transfused, the two being in apparently the same condition, and that before transfusion and within four hours of selection one of the two died.

Recipient was a colored woman, aged 35. Symptoms of pellagra developed only three weeks before transfusion, Aug. 3, 1908. There was a rapid downward progress of the patient with marked loss of weight and strength. At transfusion, the patient was unable to move her hands or head from extreme asthenia; in fact deglutition and phonation were barely possible. No distinct blood changes other than a secondary anemia were observed.

The donor was a healthy negro woman, aged 36, who had recovered from a severe attack of pellagra in the fall of 1907 .

Transfusion was performed by Crile's method, the blood flowing over from the donor to the recipient in a good stream for twenty minutes, when the donor gave signs of syncope. The wounds were closed. Both patients left their tables in good condition.

August 4: Twenty-four hours after transfusion the patient showed marked improvement mentally and in general condition.

August 7: Steady improvement continued; desquamation of the lesions was marked.

Angust 11: One week after transfusion the patient had steadily improved and walked about the ward.

There was marked gain in weight. The patient went on to a perfect recovery and to-day is about and in the same state of health as presented before the onset of the disease.

No definite conclusions from the recovery in this one case can be drawn, but we believe the coincidence to be very suggestive, considering the condition of the patient, the known inefficiency of medical therapy and the high mortality rate in this disease.

202 Conti Street.

\section{Therapeutics}

\section{PLEURISY}

The frequency of dry pleurisy during the progress of pulmonary tuberculosis is well understood, and even without definite lung lesions the cause is generally recognized and the patient consequently properly treated. Acute pleurisy with effusion, however, is all too frequently considered non-tuberculous and consequently the proper after-treatment of the disease is not carried out.

Pliysicians connected with sanatoria for the cure of tuberculosis will all attest to the great frequency of a pleurisy with effusion as preceding, by a longer or shorter length of time, a tuberculous outbreak in the lungs. It should also be stated that no condition of the lungs is so frequently entirely overlooked, or if diagnosed, improperly treated, as pleurisy with effusion. Case after case of a permanently adhesed lung with perhaps permanent cardiac displacement and with contraction of the chest, and a lower lobe of a lung that can never again be aërated, is constantly found by the lung specialist. Even if the effusion is diagnosed many a patient is told that it will gradually be absorbed, and he may even be sent into the country to rest and await its absorption, all to the permanent damage of that side of the chest. Consequently, in any pleurisy with effusion, whatever its origin, tuberculous or other, even if there is not much dyspnea or displacement of the heart, the fluid should be withdrawn if it progressively increases or if it remains several days without decreasing. It seems to be good judgement not to remove all such fluid, as the plastic serum which is in close contact with the pleural tissue apparently has the power to combat bacteria and bacterial poisons. In other words, this substance seems to contain an antitoxin, or the ability to cause antitoxin to be produced, and if it is removed the tissues become more susceptible to infection.

For the sake of a positive diagnosis of the cause of pleurisy a portion of the liquid withdrawn may be centrifuged and if tubercle bacilli are present they may be discovered. Generally, however, they are not so found even if tuberculosis is the cause of the pleurisy. A specimen of such withdrawn, settled and centrifuged, fluid which has not been allowed to become contaminated with other germs, may be injected into a guineapig or rabbit, and if tubercle bacilli are present the animal will show the disease in a certain number of weeks.

Another suggestive indication that the origin of a pleurisy is due to tuberculosis is that this centrifuged specimen of the lower stratum of the settled pleural fluid may show relatively a very large number of lymphocytes, while if the pleurisy has been caused by other infection the polymorphonuclear leucocytes are found in greatly increased numbers. Also presumptive evidence of the tuberculous or non-tuberculous origin of the pleurisy may be given by a differential blood count. If the polymorphonuclear jeucocytes are relatively increased in number, the presumption is that the condition is not tuberculous but due to some other infection. If, on the other hand, the lymphocytes are relatively increased in number and the polymorphonuclear levcocytes are not increased, the cause of the pleurisy is probably tuberculosis.

Of course diagnostic tuberculin injections and tuberculin vaccinations may be done to prove or disprove that the patient is harboring a tuberculous focus. However, it is certainly supposable, and probably true, that a patient might have a healed or quiescent tubercular lesion somowhere in him, or a tubercular gland, that to all intents and purposes was walled off from the rest of the body and inactive, and that this could be lighted up by a tuberculin injection and cause a reaction, and yet his present pleurisy be non-tubercular in origin.

Consequently, with or without a positive diagnosis of a tuberculous focus, or that the pleurisy was due to tuberculosis, the clinical evidence that a pleurisy, tuberculous or non-tuberculous, predisposes to tuberculosis of the lungs, should cause the axioms of the treatment of pleurisy with effusion to be as follows:

1. With pain or without pain in the chest, with dyspnea or without dyspnea, with cough or without cough, when suspicion is attracted to the lungs of a patient a careful physical examination should be made with the chest and back completely bared.

2. If pleural effusion is found it should not be allowed to remain in the chest more than a few days, even if there is not much dyspnea and not much displacement of the heart, unless the amount of the fluid is small and this amount is gradually diminishing.

3. It is perhaps wise not to remove all the fluid, but if the fluid rapidly reaccumulates it should certainly be again withdrawn.

4. A patient who has had pleurisy with effusion and has apparently recovered should be troated as though he were in the pretuberculous stage of pulmonary tuberculosis; in other words, he should have a longer or shorter period of rest and fresh air treatment, if possible. If such rest, during this stage of suspicior, is 